\title{
Surgical Curettage as a Treatment Modality for Alveolar Osteitis: A Wide Controversy
}

\author{
Mosaad Abdaljawwwad Abdalmawla Khalifah \\ Oral and Maxillofacial Surgery Department, Faculty of Dentistry, Kafrelsheikh University, Kafrelksheikh, Egypt
}

\section{Email address:}

mosaad_khalifa@den.kfs.edu.eg,mosaad78@hotmail.com

\section{To cite this article:}

Mosaad Abdaljawwwad Abdalmawla Khalifah. Surgical Curettage as a Treatment Modality for Alveolar Osteitis: A Wide Controversy. International Journal of Clinical Oral and Maxillofacial Surgery. Vol. 3, No. 5, 2017, pp. 26-29. doi: 10.11648/j.ijcoms.20170305.11

Received: December 2, 2017; Accepted: December 18, 2017; Published: January 8, 2018

\begin{abstract}
Dry socket is the most common post-odontectomy complication. Although blood clot disintegration is known to be the cause of the condition, agreement is lacking regarding the relative merits of various treatment methods including surgical curettage. The aim of the current study therefore was to evaluate the effectiveness of surgical curettage in a large population. A total of 506 patients were categorized into 4 groups (I, II, III, and IV) according to pain severity (mild, moderate, severe, or agonizing, respectively). All patients underwent brief saline irrigation of the socket with $2 \mathrm{ml}$ normal saline followed by curettage, irrigation with $6 \mathrm{~mL}$ normal saline and suturing. The results showed that curettage was a potent curative method for all groups. As a conclusion, curettage is a recommended treatment method.
\end{abstract}

Keywords: Alveolar, Osteitis, Curettage

\section{Introduction}

Alveolar osteitis (AO) is the most common complication of dental extraction [1]. Pain is the most important aspect of OA according to Fazakerlev and Field [2], and although a variety of treatment methods have been attempted to treat or alleviate this pain, considerable controversy exists regarding their relative efficacies [3]-[5]. One of these methods is surgical curettage. Despite curettage was considered as an acceptable treatment modality by several authors [6]-[10], it was considered as a contraindicated maneuver by others [11]-[13].

The aim of the current study was to assess whether curettage was indicated or contraindicated in alveolar osteitis in a large population of patients, utilizing definitive parameters for diagnosis and outcome assessment.

\section{Main Body}

AO treatment aims principally at pain alleviation. Surgical curettage as a treatment modality was posed with a great deal of controversy ranging from recommendation to contraindication. The aim of the current study is to investigate in depth the role of curettage. In the light of the current results, curettage is recommended. Its mechanism of action might rely on removing the diseased layer of alveolar bone in order to allow the formation of a new blood clot facing the newly formed alveolus.

\section{Methods}

The author examined patients in the Hosh Isa district (AlBehera, Egypt) who presented with pain after dental extraction during the years 2003 through 2016. A total of 506 patients diagnosed with AO ("dry socket") were included in this study. These patients were divided into 4 groups according to pain severity (Table 1).

Table 1. Pain severity levels used to assign patients to groups.

\begin{tabular}{|c|c|c|}
\hline \multicolumn{2}{|c|}{ Severity of Pain } & Description \\
\hline I & Mild & $\begin{array}{l}\text { Patients had annoying pain during most waking hours) } \\
\text { but did not need analgesics. }\end{array}$ \\
\hline II & Moderate & $\begin{array}{l}\text { Patients had pain that required and was relieved by } \\
\text { analgesics. }\end{array}$ \\
\hline III & Severe & $\begin{array}{l}\text { Patients had pain that was not relieved by analgesics } \\
\text { but that did not interfere with normal daily acitivities } \\
\text { (e.g., patients did not have to leave work and did not } \\
\text { awaken during sleep). }\end{array}$ \\
\hline IV & Agonizing & $\begin{array}{l}\text { Patients had pain that was not relieved by analgesics } \\
\text { and that interfered with normal daily activities (e.g., } \\
\text { the pain caused the patients to leave work or to } \\
\text { awaken during the night). }\end{array}$ \\
\hline
\end{tabular}


All patients underwent brief saline irrigation of the socket with $2 \mathrm{ml}$ normal saline $(0.9 \%$ solution $)$ to remove any debris. Surgery (Sur) was performed, as follows: The lamina dura and the adjacent bone layer (LDAL) were removed from the ES with a curette, and the site was irrigated with $6 \mathrm{~mL}$ warm normal $0.9 \%$ saline. Then interrupted sutures were placed across the ES without squeezing it. Sur was not just a brief curettage, even though no flaps were reflected. [Although lamina dura is a radiologic term, it was used in the current article to mean the bone layer forming this radiologic feature.]

This study complied with the Declaration of Helsinki, and the regional ethical review board of the research unit at Hosh Isa Medical Center approved the study.

\subsection{Inclusion Criteria}

1. Pain after simple dental extraction (forceps extraction)

2. Diagnosis of AO (dry socket)

3. Age 25 to 55 years.

\subsection{Exclusion Criteria}

1. Signs or symptoms of an infected socket.

2. Systemic or local conditions hindering or otherwise affecting healing.

3. Disorders causing bleeding tendencies.

4. Tooth extraction peri-menstrually.

5. Pregnancy, lactation, or use of oral contraceptives.

6. Hormonal disturbances.

7. Smoking.

The effectiveness of the treatment modality was evaluated according definitive criteria as shown in Table 2.

Table 2. Parameters for assessing treatment effectiveness.

\begin{tabular}{ll}
\hline Designation & Criteria \\
\hline \multirow{3}{*}{ Curative } & $\begin{array}{l}\text { Treatment was followed by disappearance of pain without } \\
\text { other medication (or pain became too slight to be annoying } \\
\text { or to lead the patient to seek medical or dental } \\
\text { intervention). }\end{array}$ \\
$\begin{array}{l}\text { Treatment was followed by decreased pain severity but } \\
\text { pain remained at least annoying, or treatment was followed } \\
\text { by a decrease in the dose of analgesics taken, or both. } \\
\text { Treatment was not followed by noticeable diminution in } \\
\text { pain severity (and pain remained at least annoying), or the } \\
\text { treatment had a palliative effect that was not maintained to } \\
\text { the end of a 5- minute visit. }\end{array}$ \\
\hline
\end{tabular}

The need for analgesics afterward was considered normal unless it exceeded 36 hours. The total time needed after the treatment for the patient not to seek medical or dental intervention was recorded in order to assess its effectiveness. The potency was considered high if that period was 2 days or less, moderate if it was 3 or 4 days, and low if it was 5 days or more, as the total healing period typically ranges from 7 to 10 days [14]. If the curative effect was achieved within 1 day, thus requiring only a single application, the treatment was considered a definitive therapy.

\section{Results}

The numbers of patients in groups I, II, III, and IV were 216, 172, 98 and 20, respectively. Tables 3 to 5 show the effect and potency of Sur in different groups.

Table 3. Effect of Sur in each group.

\begin{tabular}{llllll}
\hline \multirow{2}{*}{ Group } & \multicolumn{2}{l}{ Number of patients at each level of effect } & Decision: (curative/noncurative) & \multirow{2}{*}{ P values of Fisher's exact test } \\
\cline { 2 - 4 } & Curative & Palliative & Ineffective & Curative & $<0.001$ \\
\hline I & 216 & 0 & 0 & Curative & $<0.001$ \\
II & 172 & 0 & 0 & Curative & $<0.001$ \\
III & 82 & 16 & 0 & Curative & 0.0413 \\
IV & 15 & 5 & 0 & \\
\hline
\end{tabular}

Table 4. Potency of Sur in each group (Gr).

\begin{tabular}{lllllllll}
\hline Gr & 1 day & 2 days & 3 days & 4 days & 5 days & 6 days & Median & Potency \\
\hline I & 216 & 0 & 0 & 0 & 0 & 0 & 0 & 1 \\
II & 169 & 3 & 0 & 0 & 0 & 0 & 0 & High \\
III & 74 & 4 & 4 & 1 & 0 & 0 & High \\
IV & 11 & 2 & 1 & High & High \\
\hline
\end{tabular}

Table 5. Determination if Sur was a definitive curative therapy in each group.

\begin{tabular}{lllll}
\hline Group & 1 day (once) & $>$ 1 day & P value* for Fisher's exact test & Decision \\
\hline I & 216 & 0 & $<0.001$ & definitive \\
II & 169 & 3 & $<0.001$ & definitive \\
III & 74 & 8 & $<0.001$ & definitive \\
IV & 11 & 4 & 0.1184 & not definitive \\
\hline
\end{tabular}

Sur was a high potency curative treatment methods in all groups. Furthermore, it was a definitive therapy for all groups except for group IV.

\section{Discussion}

Since AO is by far the most common complication of odontectomy [1], a plethora of treatment protocols have been 
suggested to prevent and to treat this condition including surgery [6]-[10]. However, results are quite controversial [3]-[5]. Although more than one author considered curettage as a step in their treatment protocol [6]-[10], Neville et al considered curettage as a contraindicated procedures since this further increases the pain [11]. Moreover, Holmgren and Bagheri denied the alleged role of curettage in inducing bleeding and hence healing stating "this has not been shown to promote healing". As a result they contraindicated curettage [12]. Hupp contraindicated curettage hypothesizing that the condition is usually due to incomplete lysis of the blood clot; and therefore curettage removes the remaining part of the clot exposing more bone and aggravating the symptoms. The author recommended irrigation and socket dressings [13]. Therefore, the author conducted the current large prospective study investigating the role of curettage.

In order to study as homogenous group of patients as possible, the study population was restricted to otherwise healthy patients who underwent simple (forceps) extraction and did not have any known conditions affecting their healing capacities, since AO seems to reflect an interference with the healing process, resulting in blood clot loss [15]. The inclusion and exclusion criteria were determined accordingly.

To my knowledge, no published studies of AO treatment have classified patients according to pain severity or analyzed the outcome according to definitive assessment criteria. However, in the current study, patients were categorized into 4 major groups according to pain severity. The severity rating was not based on the patient's description (for example, use of words like "mild" or "severe") or on the patient's estimation on a visual pain analogue scale; rather, it relied on behavioral measures: the need for analgesics and whether the condition interfered with daily life. On assessing the outcome of treatment methods in the current study, only 3 possibilities, all well-defined, were considered. These features, in addition to the size of the population, appear unique to the current study.

In the current study, Sur (and in other words curettage) was a potent curative treatment for all groups. The lamina dura and the adjacent layer of cancellous bone were removed, and bleeding was provoked in order to re-establish a new blood clot. It seeded that removal of that layer allowed the formation of a new blood clot capable of maintaining a more secure anatomical and physiological contact with the newly formed ES. The physiologic contact might be in the form of providing the clot with the necessary circulation which was previously prevented in the case of the presence of the removed layer. If the LDAL lacks the resiliency to withstand compressive forces applied during dental extraction, ischemia in the LDAL could be inevitable, leading to formation of some sort of barrier layer between the cancellous bone in the ES and the blood clot, and in turn ultimately leading to clot disintegration and loss. In addition, release of chemicals because of ischemia might contribute to blood clot disintegration. That proposed mechanism may well relate to the role of the ES walls in maintaining the blood clot "in-situ" and providing the necessary blood supply for it. In addition, the role of ischemia seems to merit testing.

\section{Conclusion}

Dry socket results from blood clot disintegration. Until now, there has been no generally agreed on treatment of choice. Surgical curettage met a large deal of controversy. In the current large prospective study, surgical curettage was a potent curative maneuver for all groups. It seemed that the old ischemic extraction socket walls acted as a barrier layer against the blood clot, thus removal of that layer allowed for a more secure anatomical and physiologic contact with the newly-formed socket walls.

\section{Conflict of Interest Statement}

The author declares no conflict of interest.

\section{Acknowledgements}

I am deeply grateful to Dr. Barbara Gastel, Texas A\&M University, for help in refining this paper.

\section{References}

[1] A. Kolokythas, E. Olech, and M. Miloro, "Alveolar Osteitis: A Comprehensive Review of Concepts and Controversies," Int. J. Dent., vol. 2010, pp. 1-10, 2010.

[2] M. Fazakerlev and E. Field, "Dry socket: a painful postextraction complication (a review)," Dent Updat., vol. 18, pp. 31-35, 1991.

[3] S. Faizel, S. Thomas, V. Yuvaraj, S. Prabhu, and G. Tripathi, "Comparision Between Neocone, Alvogyl and Zinc Oxide Eugenol Packing for the Treatment of Dry Socket: A Double Blind Randomised Control Trial," J. Maxillofac. Oral Surg., vol. 14, no. 2, pp. 312-320, 2014.

[4] R. Alexander, "Dental extraction wound management: a case against medicating postextraction sockets," J Oral Maxillofac Surg, vol. 58, no. 5, pp. 538-551, 2000.

[5] I. R. Blum, "Contemporary views on dry socket (alveolar osteitis): a clinical appraisal of standardization, aetiopathogenesis and management: a critical review," Int $J$ Oral Maxillofac Surg, vol. 31, pp. 309-317, 2002.

[6] P. Turner, “A clinical study of 'dry socket,"” Int. J. Oral Surg., vol. 11, no. 4, pp. 226-231, 1982.

[7] G. M. Kaya GŞ, Yapici G, Savaş Z, "Comparison of Alvogyl, SaliCept patch, and low-level laser therapy in the management of alveolar osteitis," J Oral Maxillofac Surg, vol. 69, pp. 1571-1577, 2011.

[8] A. G. Halberstein RA, "Clinical management and control of alveolalgia ('dry socket') with vitamin C," Am J Dent, vol. 16, pp. 152-154, 2003.

[9] T. O. Ogunlewe MO, Adeyemo WL, Ladeinde AL, "Incidence and pattern of presentation of dry socket following nonsurgical tooth extraction," Nig $Q J$ Hosp Med, vol. 17, pp. 126-130, 2007. 
[10] G. C. Taberner-vallverdú M, Nazir M, Sánchez-garcés Á, "Efficacy of different methods used for dry socket management: A systematic review," vol. 20, no. 5, pp. 633639, 2015.

[11] Neville BW, Damm DD, Allen CM, and Chi AC, Oral \& Maxillofacial Pathology, 4th ed. Missouri: Elsevier, 2016.

[12] Holmgren EP and Bagheri SC, "Alveolar osteitis (Dry socket)," in Clinical Review of Oral and Maxillofacial Surgery A Case-Based Approach, 2nd ed., Bagheri SC, Ed. Missouri: Elsevier (Mosby), 2014, p. 126.
[13] Hupp JR, "Prevention and management of extraction complication," in Text Book of Contemporary Oral and maxillofacial surgery, 6th ed., Hupp JR, Ellis E, and Tucker MR, Eds. Missouri: Elsevier (Mosby), 2014, pp. 174-187.

[14] G. G. Gowda, D. Viswanath, M. Kumar, and D. Umashanker, "Dry Socket (Alveolar Osteitis): Incidence, Pathogenesis, Prevention and Management," J. Indian Acad. Oral Med. Radiol., vol. 25, no. 3, pp. 196-199, 2013.

[15] H. Birn, "Etiology and pathogenesis of fibrinolytic alveolitis('dry socket')," Int J Oral Surg, vol. 2, pp. 215-263, 1973. 